\title{
Performance of Profiled Single Noise Barriers Covered with Quadratic Residue Diffusers
}

\author{
M. R. Monazzam and Y. W. Lam \\ School of Computing, Science and Engineering, \\ University of Salford, Salford, M5 4WT, UK
}

\begin{abstract}
The paper describes an investigation about the acoustic performance of noise barriers with quadratic residue diffuser (QRD) tops, and with T-, Arrow-, Cylindrical and Y- shape profiles. A 2D Boundary Element Method (BEM) is used to calculate the barrier insertion loss. The results of rigid and with absorptive coverage are also calculated for comparisons. Using $Q R D$ on the top surface of almost all barrier models presented here is found to improve the efficiency of barriers compare with using absorptive coverage at the examined receiver positions. T-shape and Arrow-shape barriers are also found to provide better performance than other shapes of barriers. The best shape of barriers for utilising QRD among the tested models is the T-shape profile barrier. It is found that reducing the design frequency of QRD shifts the performance improvement towards lower frequency, and therefore the most efficient model for traffic noise is a barrier covered with a QRD tuned to around $400 \mathrm{~Hz}$
\end{abstract}

\section{Keywords: Noise barrier; Diffusion; Boundary Element}

\section{Introduction}

Noise barriers are one of the most common methods for environmental as well as industrial noise control. The control of traffic noise from highways, railroads, airports and other outdoor noises from machinery and other industrial sources are some examples of its application.

Basically a barrier prevents sound waves from reaching a listener in the shadow zone by the direct path. Sound can only reach the listener by other indirect ways, usually through diffraction over the barrier top. There are other ways that will affect the propagation of sound to the receiver behind the barrier, such as atmospheric refraction, turbulence scattering and 
diffraction around the vertical sides of a finite length barrier. In this paper these effects will be ignored and we will focus on the diffraction over the barrier top in a still, homogeneous atmosphere.

There are a number of methods that can be applied to improve the efficiency of barriers, such as increasing a barrier's height, utilising sound absorbing materials and different profiles at the top edge. The performance of noise barriers with different top profiles has been investigated by many researchers. Hothersall et al [1] reviewed the works done on barriers with caps having T, Y, and arrow profiles. Crombie et al. [2] introduced multiple-edge noise barriers, which could increase their efficiency in the deep shadow zone. An approximate analytical method for calculating the diffraction field behind a jagged edge noise barrier idealised as a thin rigid half- plane having an irregular edge, was presented by Menounou et al. [3].

A numerical study of the insertion loss (IL) of some rectangular, T-shaped and cylindrical edged noise barriers with rigid, absorbing and soft surfaces has been carried out by Fujiwara et al. [4]. In their study, they found that the most efficient design was a T-shape with a soft upper surface. However it was also found that a uniform series of wells in the upper surface of a T-shape barrier could produce insertion loss values equal to those of a soft surface over a significant range of frequencies.

In practice, using absorbent material on barrier is not always practical due to environmental variables especially close to highway. Their efficiency decreases after a short time because typical fibrous/porous absorptive materials are usually sensitive to environmental contaminations caused by rain, dust, and so on. Air contaminants like dust, mist, fog and so on can destroy the effectiveness of the material. Due to the above reasons, barrier designers are looking for alternative methods that can provide high performance but are less dependent on environmental factors.

\section{Quadratic Residue Diffuser}

Pseudo-stochastic diffusers, which were invented by Schroeder, are one or two- dimensional periodic surface structures, composed of wells with different depths that are intended to reduce specular sound reflection by scattering the incident sound energy in to a wide range of 
directions. Within one period, the depths of the wells vary according to a pseudo-stochastic number sequence or pattern. In each well, the incident wave will excite a pressure wave travelling toward the rigid bottom from which it is reflected. After returning to the entrance plane of the structure, theses waves will have undergone different phase shifts corresponding to the different path lengths they have travelled.

If the phase differences are sufficiently large, the structure will produce a significant scattering of reflected wave, with scattering characteristics depending on the depth sequence of the elements. The most popular Schroeder diffuser is the quadratic residue diffuser (QRD), which employs the quadratic residue sequence to determine the well depths. Ideally a QRD should produce a uniform scattered field within its design frequency range.

This paper investigates the introduction of a new design of barrier using diffuser on the upper surface of a single barrier. In this report the performance of noise barriers with QRD profile is calculated using the two-dimensional boundary element method. Insertion loss spectra at 1/15-octave centre frequencies are calculated. The results are compared with barrier with either rigid or absorptive top surface.

\section{Numerical Modelling Method}

The situation for which predictions are to be applied is shown in Fig. 1. In this model only two dimensional (2D) cases are considered. The $\mathrm{x}$-axis lies in the ground plane and $y$-axis is normal to the ground plane, $\gamma$ denotes the surface of the cross-section of the barrier above the ground plane. A uniform line source (with $\mathrm{e}^{-\mathrm{i} \omega \mathrm{t}}$ time dependence) is at $\mathbf{r}_{0}=\left(\mathrm{x}_{0}, \mathrm{y}_{0}\right)$ and $\mathrm{p}\left(\mathbf{r}, \mathbf{r}_{0}\right)$ is the acoustic pressure at $\mathbf{r}=(\mathrm{x}, \mathrm{y})$. A noise barrier of infinite length lies on the plane, and it is assumed that the acoustical properties and the cross-section shape of the noise barrier do not vary across its length. Therefore the problem is two-dimensional, with the z-axis parallel to the barrier length, and the geometrical and acoustical variables constant in the z-direction. The barrier surface is assumed to be locally reacting with specific surface admittance $\beta\left(\mathbf{r}_{\mathrm{s}}\right)$ at point $\mathbf{r}_{\mathrm{s}}=\left(\mathrm{x}_{\mathrm{s}}, \mathrm{y}_{\mathrm{s}}\right)$ on $\gamma$, where $\mathbf{r}_{\mathrm{s}}$ is a location vector of a point on $\gamma$.

The Helmholtz wave equation is solved by the boundary integral equation at a single frequency using the boundary element method. 
The surface of the barrier is divided into a number of straight line elements $\gamma_{1}, \gamma_{2}, \ldots, \gamma_{\mathrm{n}}, \ldots, \gamma_{\mathrm{N}}$. The acoustic pressure, $\mathrm{p}\left(\mathbf{r}_{\mathrm{n},}, \mathbf{r}_{0}\right)$ is assumed to be constant over each element and is calculated at the mid-point $\mathbf{r}_{\mathrm{n}}$, where $\mathbf{r}_{\mathrm{n}}$ is the location vector of the mid-point of the nth element. Thus the approximation of the integral equation for the rigid ground over an admittance surface reduces to $[4,5]$ :

$$
\varepsilon(\mathbf{r}) p\left(\mathbf{r}, \mathbf{r}_{0}\right)=G\left(\mathbf{r}_{0}, \mathbf{r}\right)+\sum_{n=1}^{N} p\left(\mathbf{r}_{n}\right) \int_{\gamma}\left(\frac{\partial G\left(\mathbf{r}_{\mathrm{s}}, \mathbf{r}_{\mathbf{n}}\right)}{\partial n\left(\mathbf{r}_{\mathrm{s}}\right)}-i k \beta\left(\mathbf{r}_{\mathrm{s}}\right) G\left(\mathbf{r}_{\mathrm{s}}, \mathbf{r}\right)\right) d s\left(\mathbf{r}_{\mathrm{s}}\right)
$$

where ds $\left(\mathbf{r}_{\mathrm{s}}\right)$ represents the length of an element of $\gamma$ at $\mathbf{r}_{\mathrm{s}}, \mathrm{k}$ is the wave number and $\partial / \partial \mathrm{n}\left(\mathbf{r}_{\mathrm{s}}\right)$ denotes the partial derivative in the direction of the normal to $\gamma$ at $\mathbf{r}_{\mathrm{s}}$ directed outward into the propagation medium. $\varepsilon(\mathbf{r})=1$ when $\mathbf{r}$ lies anywhere in the propagation medium except on $\gamma$; $\varepsilon(\mathbf{r})=0.5$ if $\mathbf{r}$ is a point on $\gamma$ which is not a corner point. However if $\mathbf{r}$ is a corner point then $\varepsilon(\mathbf{r})=\Omega /(2 \pi)$, where $\Omega$ is the angle in the medium subtended by the two tangents to the boundary at $\mathbf{r}$. $G\left(\mathbf{r}, \mathbf{r}_{\mathbf{0}}\right)$ is the acoustic pressure at $\mathbf{r}$ due to the source at $\mathbf{r}_{0}$ when propagation is taking place above a plane rigid ground with no barrier present. $G\left(\mathbf{r}, \mathbf{r}_{0}\right)$ is written as [4]:

$$
G\left(\mathbf{r}, \mathbf{r}_{0}\right)=-\frac{i}{4}\left\{H_{0}^{(1)}\left(k\left|\mathbf{r}_{0}-\mathbf{r}\right|\right)+H_{0}^{(1)}\left(k\left|\mathbf{r}_{0}^{\prime}-\mathbf{r}\right|\right)\right\}
$$

where $\mathbf{r}_{0}{ }^{\prime}=\left(\mathrm{x}_{0},-\mathrm{y}_{0}\right)$ is the image of the source in the ground, $\mathrm{H}_{0}{ }^{(1)}$ is the Hankel function of the first kind of zero order.

By putting $\mathbf{r}$ equal to $\mathbf{r}_{\mathrm{m}}$ for $\mathrm{m}=1,2, \ldots ., \mathrm{N}$ in equation (1) a set of linear equations is obtained which can be used to solve the solution of the surface pressure $p\left(\mathbf{r}_{n}, \mathbf{r}_{0}\right)$. Equation (1) can then be used to calculate the pressure at a receiver point in the propagation medium. This formulates the pressure at a point, as a combination of the direct pressure from the source and a surface integral of the pressure and its derivative over the barrier surface.

In the numerical simulations, dimension of elements was taken to be less than $\lambda / 5$ to give a reasonable representation of constant surface pressure over an element [6]. The QRD is represented by a box with the top surface having an admittance distribution as given by the simple phase changes due to plane wave propagation within the QRD wells. It should be noted that this assumption is not accurate above the upper cut-off frequency of the QRD when plan wave propagation breaks down. However it is considered an acceptable assumption for this investigation which is primarily aimed at evaluating the effect of 
diffusive/reactive boundary on barrier performance. This assumption will be validated later on one of the QRD barriers to confirm the result.

For the simulation of the effect of absorbent surfaces, a fibrous materials is assumed and the empirical formulae of Delany and Bazley [7] are used for the calculation of the characteristic impedance $\mathrm{Z}_{\mathrm{ch}}$ and propagation constant of the fibrous material. Note that this approach is different from Ref.[4]'s assumption of an ideal specific normal surface impedance of 1 for a perfectly absorbing surface. Here it is considered that using a typical fibrous cover is more realistic than assuming the ideal absorptive condition.

In order to avoid interference between the source and its ground image the sound source is placed on a rigid ground $5 \mathrm{~m}$ from the barrier, i.e. located at coordinate $(5,0)$, in all cases. The result is then free from ground interference patterns when the receiver is also on the rigid ground. The acoustic pressure is calculated at 1/15-octave centre frequencies between 50 and $4000 \mathrm{~Hz}$ at different receiver locations. The insertion loss at each frequency is calculated as:

$$
I L=-20 \log _{10}\left|\frac{p\left(\mathbf{r}, \mathbf{r}_{0}\right)}{G\left(\mathbf{r}, \mathbf{r}_{o}\right)}\right| d B
$$

Note that because of the mirroring effect of the ground, the $I L$ calculated by Equation (3) will be about $6 \mathrm{~dB}$ lower than the attenuation of an equivalent semi-infinite barrier (no ground) under the consideration of geometrical diffraction theory. This is because there are now 4 identical diffracted paths over the barrier that contribute to the sound pressure at the receiver due to the reflecting ground, while in the absence of the barrier there will only be 2 contributing paths - the direct and reflected paths. An illustration of the situation can be found in Figure 1, and Equations (2) and (3) of Ref.[8].

\section{Results}

The performance of a few different shapes of single noise barriers with different upper edge conditions has been predicted using 2D-boundary element method. The designs used in the simulations are shown in Fig. 2.

The overall height of all type of barriers is fixed at $3 \mathrm{~m}$, which is the same as that used in Ref.[4]. The stem and cap thickness is respectively 0.1 and $0.3 \mathrm{~m}$ in all models. The width of 
the $\mathrm{T}$ top in $\mathrm{T}$-shape barrier is $1 \mathrm{~m}$, diameter in Cylinder is $1 \mathrm{~m}$ and the width of each side of the caps in both Arrow and Y-shape is $1 \mathrm{~m}$. The angle of the arrow shape barrier is 60 degrees while the angles for "Y" shape barrier are 30 and 60 degrees.

The above dimensions are similar to those used in previous studies [1,2,4]. Three different surfaces were used on the barrier including rigid, absorbing and QRD coverage.

I. Rigid surface: All surface admittances are zero, which is the Neumann boundary condition.

II. Absorbing surface: Only the upper surface of the cap is covered with fibrous absorptive material. A typical flow resistivity of $20000 \mathrm{Ns} / \mathrm{m}^{4}$ is assumed for the fibrous material. The thickness of the fibrous material is fixed at $0.2445 \mathrm{~m}$ (the same as the thickness of the QRD, see below). Barrier models with this absorptive cover are labelled with a prefix " $A$ " in this paper. For example a T-shape barrier with the absorptive cover is labelled as ATshape barrier.

III. QRD barrier: Quadratic residue diffusers with different designs are fixed to the upper surface of barriers shown in Fig. 2 with the overall height remained constant. Different designs are used to examine how diffusers affect the performance of barriers. The different designs and their model names are given in Table 1.

The dimension of one of the tested QRD designs (labelled model "A” here) is shown in detail in Fig. 3. All other models are fitted in the same overall frame, so that the overall lengths of all the QRD configurations used in different barrier models are the same. The maximum well depth among all the QRD models, which corresponds to a QRD tuned at $400 \mathrm{~Hz}$, is $0.2445 \mathrm{~m}$. This maximum depth was the same as the absorbent material thickness used in the last section.

In order to investigate to what extent the QRD barriers improve the performance of different forms of barriers, the results are compared against a reference barrier, which is chosen to be the $\mathrm{T}$ shape barrier with the fibrous absorptive cover (labelled as AT shape barrier). For convenience this model will also be referred as the "Ref" model.

The following equation is used to find the well depth regarding to design frequency $[9,10]$, 
$d_{n}=\frac{c\left(n^{2} \bmod N\right)}{N\left(2 f_{r}\right)}$

where $\mathrm{n}$ is an integer, $N$ is an odd prime number and $f_{r}$ is the design frequency. Table 1 shows the configurations of the different QRD barrier models. In this table under the column "Sequence", the sign " 1 " is for a sequence of 0142241 and the sign of "- 1 " is for an inverse sequence.

The lower and upper wavelength limits can also be approximated roughly by [10]:

$$
\lambda_{\max } \approx \frac{2 N d_{\max }}{n_{\max }} ; \lambda_{\min } \approx 2 w
$$

where $d_{\max }$ is the maximum depth and $n_{\max }$ is the maximum number from the sequence $\left(n^{2} \bmod N\right)$. Therefore the frequency bandwidth for a N=7 QRD with well width of $12 \mathrm{~cm}$ is from $f_{r}$ to about $1.4 \mathrm{kHz}$, e.g. for a $\mathrm{N}=7$ QRD with maximum depth of $0.2445 \mathrm{~m}$ and a well width of $12 \mathrm{~cm}$ the frequency bandwidth should be from 400 to $1.4 \mathrm{kHz}$. In practice the frequency bandwidth is slightly wider than this.

In all calculations in this paper the fin thickness is assumed to be negligible. If the surfaces of the wells are rigid and it is sufficiently wide for viscous and thermal condition to be negligible then the specific input admittance at the open side of the channel can be represented approximately as:

$\beta=-j \tan (k d)$

where $k$ is the wave number and $d$ is the well depth.

\section{a. Whole surface modelling}

As it was mentioned earlier in this paper the main BEM calculation is based on the assumption that the QRD can be represented by box with a variable impedance surface. The accuracy of this assumption is determined by comparing a prediction using this assumption with a prediction that model the exact geometry of the QRD, which is referred to as "whole surface modelling” here. The comparison is made on the most frequent used barrier in this study, model "G", in Fig. 4. In this model the fin thickness is $5 \mathrm{~mm}$. Apart from the fins, the size of elements remains the same as in the box approximation method. As expected, the result shows very good agreement up to just below the upper cut off frequency, which is 
$1.4 \mathrm{kHz}$ in this case. Above the upper cut off frequency, i.e. above the design frequency range of the QRD, the overall trends of the 2 predictions are still similar but the box approximation gives rise to noticeable error, which can be about $5 \mathrm{~dB}$ at certain single frequencies. With this in mind, the box approximation is a convenient and accurate representation for the investigation of QRD barrier performance within and under the design frequency range of the QRD.

\section{b. QRD edged barriers of different shapes}

In order to give a clear demonstration of the results for different basic barrier shapes, the four models, G, O, M and R are compared with their original rigid and absorbent shapes. These four models have the same QRD design, with the same frequency design, which is $400 \mathrm{~Hz}$, and the same well width of $12 \mathrm{~cm}$. In other words the QRDs used in these models have the same frequency bandwidth (from about $400 \mathrm{~Hz}$ to $1.4 \mathrm{kHz}$ ), and the only difference is the shape of the barrier top that the QRDs are fitted into.

Employing the designed QRD on model "G” (T-shape) increases significantly the insertion loss of the barrier compared with both rigid and absorbent T-shape profiles at frequencies above $315 \mathrm{~Hz}$. Fig. 5.a clearly shows the peaks of insertion loss gained by model "G" at 315,630 and $1000 \mathrm{~Hz}$. Increases at $400 \mathrm{~Hz}, 1.25 \mathrm{kHz}$ and $2 \mathrm{kHz}$ are also significant. At frequencies lower than $315 \mathrm{~Hz}$ and above $2 \mathrm{kHz}$ (outside the QRD frequency bandwidth) the performance start to decline and go even slightly lower than rigid shape at very low frequencies. It is worth noting that only one QRD period is used in this model.

In Fig. 5.b two identical QRD periods $\left(f_{r}=400 \mathrm{~Hz}, w=0.12 \mathrm{~m}\right)$ are used on the cylinder profile. In this model the first significant peak is at $315 \mathrm{~Hz}$, the second and third visible peak $630 \mathrm{~Hz}$ and $1.25 \mathrm{kHz}$ respectively.

Utilising two identical QRDs $\left(f_{r}=400 \mathrm{~Hz}, w=0.12 \mathrm{~m}\right)$ on an Arrow-shape barrier significantly improves the performance compared with the corresponding rigid and even the absorptive arrow shape barriers as one can see in Fig. 5.c. There are successive peaks in the frequency spectrum that push the performance of the model " $M$ " barrier significantly higher than the other two in the mid frequency ranges. The first maximum is at $250 \mathrm{~Hz}$ and the second is at 
$315 \mathrm{~Hz}$. The peaks at $1 \mathrm{kHz}$ and $1.25 \mathrm{kHz}$ are also significant. As expected at frequencies higher than $1.6 \mathrm{kHz}$ (above the upper cut-off frequency of the QRD) the QRD barrier becomes less efficient, but the absorbent barrier is still significantly better than that of rigid shape barrier.

On the Y-shape barrier employing two identical QRDs doesn't show significant improvements. As it is shown in Fig. 5.d, although two peaks at $250 \mathrm{~Hz}$ and $1 \mathrm{kHz}$ are higher than the absorbent Y- shape the trends of all three Y-shape models are very close to each other in the entire frequency spectrum. This is because the QRDs and likewise the absorptive surfaces are not facing the source and receiver.

A common effect of the QRDs on all the different barrier models is that the barrier IL becomes more frequency dependent compared with the rigid and absorbent equivalents. There are significant peaks occurring in the frequency spectrum. This is consistent with the operational characteristics of a QRD in which well resonances occur at various frequencies corresponding to the well depth sequence. The best improvement is seen within the design frequency bandwidth of the QRD from about $315 \mathrm{~Hz}$ to $1.4 \mathrm{kHz}$, with also noticeable improvements at $2 \mathrm{kHz}$ in model " $\mathrm{G}$ " and at $250 \mathrm{~Hz}$ in model "S". Every models show a significant peak at $1 \mathrm{kHz}$. In all cases the employment of QRD improves the performance of the barriers within the design frequency bandwidth of the QRD.

\section{c. Barrier configurations with different $Q R D$ designs}

The calculated spectra of insertion loss of all tested models with a T- shape profile are shown in Fig. 6. For clarity the results are split to four graphs. Fig. 6.a, b, and c have QRDs with prime number 7 , and show performance variations due to frequency design and different well width. Within each subplot the design frequency is the same but the well width is reduced from $12 \mathrm{~cm}$ to $6 \mathrm{~cm}$ and then $2 \mathrm{~cm}$. This results in higher upper cut-off frequencies and larger numbers of QRD periods for the smaller well widths. The design frequencies are $1 \mathrm{kHz}$, $500 \mathrm{~Hz}$ and $400 \mathrm{~Hz}$ respectively for the QRDs in Figures 6.a, 6.b and 6.c. The effect of reducing the prime number is shown in Fig. 6.d, in which model " $K$ " has a QRD with $N=5$ and a well width of $18 \mathrm{~cm}$ on top of the T- shape barrier. 
There are common trends in Fig. 6.a, b and c, which are related to the design frequency and the well width of the QRD. It is clear that lowering the design frequency of the QRD shifts the first peak of the insertion loss of the QRD barrier to a lower frequency. Indeed the frequency of the first peak is very close to the design frequency, again evidence that the peaks in the insertion loss are strongly related to the characteristic resonances of the QRD wells. The effect of decreasing the well width is not as clear. In theory a smaller well width gives a higher upper cut-off frequency to the QRD and therefore should result in better performance at higher frequencies. This is not shown by this simulation. This is a limitation of the current assumption used in the numerical modelling in which the QRD is approximated by a box with surface admittance distribution given by the simple plane wave admittance of the QRD wells. This approximation is maintained even at frequencies above the QRD's upper cut-off frequency above which the plane wave assumption is no longer adequate. Hence results above the cut-off frequencies $(1.4 \mathrm{kHz}$ for $w=12 \mathrm{~cm}, 2.8 \mathrm{kHz}$ for $w=6 \mathrm{~cm}$, and $8.4 \mathrm{kHz}$ for $w=2 \mathrm{~cm}$ ) may not be accurate, as already been discussed earlier under Figure 4 . Below the upper cut-off, a decrease in well width sometime increases the performance of the barrier at peak points, however the efficiency is decreased at the minimum points with the overall effect that the broadband mean insertion loss remained roughly unchanged. It should be reminded that viscous and thermal loses were ignored in the calculation of the QRD admittance. For very small well widths, these effects could be significant and introduce larger impedance as well as significant absorption [11, 12].

In Figure 6.d, the reduction in the QRD prime number changes the maximum and minimum frequencies over which the QRD is effective. The frequency at which the barrier with QRD with $\mathrm{N}=5$ starts to become effective is $250 \mathrm{~Hz}$ and the second and the third peaks appears at 1 $\mathrm{kHz}$ and $1.6 \mathrm{kHz}$. More importantly, Fig 6.d clearly shows that a QRD with a smaller prime number $\mathrm{N}$ has less peaks in the insertion loss spectrum. With a smaller $\mathrm{N}$ the number of different well depths is reduced. As a consequence the number of well resonances, and hence the insertion loss peaks, is also reduced. In Figure 6.d, although the increases in insertion loss at the peaks at $315 \mathrm{~Hz}, 1 \mathrm{kHz}$ and $1.6 \mathrm{kHz}$ are significant, the insertion loss in between is no more than that provided by the equivalent rigid T-shape barrier. The result strongly suggests that a QRD with a larger variation of well depths (larger N) will perform better when applied to noise barriers. 
Using two QRDs on two different configurations of Y-shape barrier with, 30 and 60 degrees of the $\mathrm{Y}$-angle, made the two barrier models "R" and "S". Their performances are compared with the T- shape barrier in Fig. 7. Increase the angle from 30 to 60 degrees could raise the gained insertion loss between $200 \mathrm{~Hz}$ and $3.15 \mathrm{kHz}$, although at frequencies bellow $200 \mathrm{~Hz}$ the situation is the opposite. Overall the 60 degrees Y-shape barrier performs better with the QRD than the 30 degrees barrier, which is not surprising since a larger Y-angle exposes larger portion of the QRD surface to the source and receiver.

\section{d. QRD barrier top compared with absorptive top}

According to Hothersall et al. [1] T-shape profile barriers with strongly absorbent materials on the top surface of the $\mathrm{T}$ - part was the best choice among barrier design he investigated. Fig. 8 and 9 compare the new QRD barrier designs in this research with the equivalent absorbent T-shape barrier, labelled "Ref" in the figures. In all cases the barriers have the same overall height, thickness, source, receiver and ground conditions. Significant improvement is provided by model " $G$ " ( $T$ - shape profile with one period of the $N=7$, $f_{r}=400 \mathrm{~Hz}$ QRD) at frequencies above $315 \mathrm{~Hz}$ as shown in Fig. 8. The other two QRD models also improve the efficiency of the barrier but at higher starting frequencies because of their higher design frequencies.

The A- weighted road traffic noise spectrum [13] is calculated by combining the results for insertion loss at one-third octave band centre frequencies over the range 50 to $4000 \mathrm{~Hz}$ and assuming a suitable source spectrum. It is usually done to average the interference effects observed at single frequencies, and allow smoother trends to be identified more easily. It is worth adding that the result in 1/15 octave band was averaged to give 1/3-octave band based on simple energy averaging method. At receiver point $(50,0)$ the A- weighted road traffic noise is 14.5, 15.8 and $15.9 \mathrm{~dB}(\mathrm{~A})$ for model " $\mathrm{A}$ ”, " $\mathrm{D}$ ” and "G” respectively, compared with $15.7 \mathrm{~dB}(\mathrm{~A})$ for the "Ref" barrier. Model "G" enhances the mean insertion loss by a further $0.2 \mathrm{~dB}(\mathrm{~A})$ compared with an absorbent T- shape barrier at this receiver point.

The effect of the QRD on an Arrow shape barrier (model "M") is also compared with the reference absorbent $\mathrm{T}$ shape barrier in Fig. 9. The influence of the QRD on an Arrow shape barrier (model "M") is stronger than on a T-shape barrier (model "G”) in the frequency range between $250 \mathrm{~Hz}$ to $2 \mathrm{kHz}$, but worse at frequencies below $250 \mathrm{~Hz}$. 


\section{e. IL difference of QRD top barriers with Reference barrier}

A calculation has been carried out to find the increase in the barrier insertion loss, $\Delta I L$, due to the application of the QRD to show the domain of improvement of selected models compare to the absorbent T- shape "Ref" barrier. In each frequency the insertion loss of the selected model is subtracted by that of the "Ref" model. Fig. 10 and 11 show the results.

While the effect of model "G" (T-shape) is mainly between $315 \mathrm{~Hz}$ to $1.25 \mathrm{kHz}$, the effective frequency domain of model " $M$ ” (Arrow-shape) is extended one 1/3 octave band below and above that of model "G". Outside the effective frequency domain the insertion loss in model " $G$ " is about the same as that of an equivalent absorbent T-shape barrier. However the reduction at frequencies below $250 \mathrm{~Hz}$ is rather significant in model "M".

Fig.11 shows a clear view of the effect of different frequency designs of QRD on the barrier models compared to the "Ref” absorbent T- shape barrier. All three QRD models improve the efficiency of the barrier significantly within their respective frequency bandwidths. Decrease in the design frequency shifts the effective frequency domain towards lower frequencies. So the first effective frequency for model " $\mathrm{A}$ ” $\left(f_{r}=1 \mathrm{kHz}\right)$ is $630 \mathrm{~Hz}$ while that for model " $\mathrm{D}$ " $\left(f_{r}\right.$ $=500 \mathrm{~Hz})$ is $315 \mathrm{~Hz}$ and finally $250 \mathrm{~Hz}$ is the starting effective frequency for model " $\mathrm{G}$ ” ( $f_{r}$ $=400 \mathrm{~Hz})$.

\section{f. Broad band insertion loss}

The broadband insertion loss at nine receiver positions 20, 50 and $100 \mathrm{~m}$ from the centre line of the barrier on the ground and at 1.5 and $3 \mathrm{~m}$ above the rigid ground for the A-weighted traffic noise spectrum [13] are shown in Table 2. The insertion loss over the receiver positions for each barrier and the $\Delta \mathrm{IL}$, which is the difference in the mean insertion loss relative to the reference absorbent T-shape barrier are also shown. Using QRD on the Arrowshape and cylindrical shape barrier (model "O") could not improve the A- weighted mean insertion loss of the barriers compare to the reference barrier. Different forms of the $\mathrm{Y}$-shape barrier, either absorbent or QRD tops (models “R” and “S”), have slightly higher mean insertion loss than the reference absorptive T-shape model. 
It is worth noting that employing a QRD with $f_{r}=400 \mathrm{~Hz}$ on both T-shape and Y- shape raises the mean insertion loss of all barrier shapes (except Model "I") beyond that of the "Ref" absorbent T-shape barrier. Using this QRD tuned to $400 \mathrm{~Hz}$ on a rigid T-shape barrier (model “G”) improves the efficiency of the barrier by $0.9 \mathrm{~dB}(\mathrm{~A})$ compared with that of the reference absorbent T-shape barrier.

Reducing the frequency design of the QRDs from $1 \mathrm{kHz}$ (model “A”) to $500 \mathrm{HZ}$ (model “D”) and even lower down to $400 \mathrm{~Hz}$ (model "G”) improved the A- weighted insertion loss of barriers from $16.6 \mathrm{~dB}(\mathrm{~A})$ in model “A” to $17.7 \mathrm{~dB}(\mathrm{~A})$ in model "D” and $19 \mathrm{~dB}(\mathrm{~A})$ in model "G”, which could be explained by the shifting of the frequency effect to low frequencies by QRDs with lower frequency design.

Although reducing the well width of the applied QRDs could potentially increase the absorption coefficient of the device [12], it is shown that it has negative effect on the Aweighted insertion loss of any $\mathrm{T}$ - shape QRD barriers with different frequency designs (except model "C" with a slight improvement). In this case reducing the well width in a QRD tuned to $400 \mathrm{~Hz}$ from $12 \mathrm{~cm}$ in model "G" to $2 \mathrm{~cm}$ in model "I" decreased the Aweighted insertion loss of the barriers from $19 \mathrm{~dB}(\mathrm{~A})$ to $16.6 \mathrm{~dB}(\mathrm{~A})$ respectively.

In fact in all types of barriers investigated both absorbent material and QRD (apart from model "O" at $20 \mathrm{~m}$ distance from barrier) can improve the efficiency of the barrier compare to their corresponding rigid shapes on the ground, as shown in Table 3. However the amount of increase is dependent on the profile of the barriers. For example while covering the top surface of Arrow-shape barrier with QRD improves the mean insertion loss by $2.9 \mathrm{~dB}(\mathrm{~A})$ at all receiver positions, the improvement for a Y-shape barrier is only about $2 \mathrm{~dB}(\mathrm{~A})$. In Tshape barrier the improvement provided by the $\mathrm{QRD}$ is higher than that provided by the absorbent material. It should also be noted that the QRDs used in the investigation are nonabsorptive. It will be an interesting further work to investigate if an absorptive QRD, as suggested in References [11, 12], can further improve the performance of a QRD barrier.

Furthermore as one can also see from Table.3 that the T- shape profile, with improvement between 0.9 to $1 \mathrm{~dB}(\mathrm{~A})$ due to $\mathrm{QRD}$ compare to its equivalent absorptive configurations, is the best form to use QRD on. In contrast the Cylinder - shape profile, with between 0.5 to 1.4 $\mathrm{dB}(\mathrm{A})$ deterioration compare to its absorptive equivalent, is the worst shape to employ QRD. 


\section{g. Sound field at the shadow zone}

The details of the far and near field effect of the presented models are shown in Figures 12 to 13 in contour plots of the broadband differences of A-weighted insertion loss relative to the "Ref” absorptive T-shape barrier and absorbent Arrow - shape at 400 receiver positions for two selected models: QRD barrier model “G” and “M”. Fig. 12 shows that model “G” (QRD on T-shape) has got better performance in two distinctive areas of very close to the ground and the field beyond $10 \mathrm{~m}$ from the barrier where the mean insertion loss increase is notably higher at longer distances with higher height. The amount of improvement in the far field at the height of 6 to $8 \mathrm{~m}$ can reach up to 2.5 to $3.5 \mathrm{~dB}(\mathrm{~A})$, while the same amount of improvement is visible at zones close to the ground in both near and far field. Close to the barrier, especially at high receiver heights, the improvement is small to negative. This is probably due to more sound being diffracted upwards by the QRD, which also explains the higher insertion loss in the far field receiver and lower heights.

Fig. 13 shows that the performance of model "M" (QRD on Arrow-shape) is higher than its equivalent absorbent Arrow - shape in the far filed at the height above $3 \mathrm{~m}$. It produces 1.5 to $2 \mathrm{~dB}$ (A) higher insertion loss than the absorbent Arrow-shape barrier in the far field and height of 6 to $8 \mathrm{~m}$, similar to what is seen for model "G" in Fig. 12. Unlike model "G" the improvement made by QRD on Arrow shape profile is very small to negative close to ground. The area of negative effect is widened at the far field.

\section{Discussion and conclusion}

The attenuation of sound by QRD edged single noise barriers has been investigated using a two-dimensional boundary element model. Broadband insertion loss has been predicted over a range of representative receiver positions using a A-weighted traffic noise spectrum in 1/15-octave band from $50 \mathrm{~Hz}$ to $4000 \mathrm{~Hz}$. The performance of three different top surfaces; rigid; absorptive; and QRD on four different types of barriers; T-shape; Cylindrical; Arrowshape; and Y-shape has been evaluated. The performances of QRD barriers have been compared with their equivalent absorbent and rigid barriers, as well as compared with a 
reference absorbent T- shape profile (the "Ref" model). The results can be summarised as follows:

i. As already described in previous works, utilising absorbent material on the top surface of a barrier could improve the efficiency of barriers. The best profile for using absorption was found here to be the Arrow-shape with a $2.9 \mathrm{~dB}(\mathrm{~A})$ mean insertion loss improvement on the ground $50 \mathrm{~m}$ far from barrier. This was explained by the high proportion of the treated surfaces facing the source and receiver in this barrier profile

ii. Every single QRD barrier could increase the performance of rigid barrier within certain frequency domain depending on its frequency design. It is possible to shift the frequency effect to higher or lower frequencies by changing the utilized QRD frequency design.

iii. Utilising QRD on different barrier profiles was found to produce insertion loss that varies significantly with frequency and is strongly dependent on the design frequency of the QRD. Overall, the numerical simulations in this study have shown that using QRD to cover the top surface of T- shape and Y- shape barrier produces higher barrier A-weighted insertion loss than using a typical fibrous absorbent material on the barrier.

iv. Among the three different design frequencies tested, namely 1000, 500 and $400 \mathrm{~Hz}$, the most efficient design was found to be a QRD tuned to $400 \mathrm{~Hz}$. As expected, lowering the design frequency while keeping the upper cut-off frequency constant provided higher broadband mean insertion loss.

v. It is found that putting one QRD with a design frequency of $400 \mathrm{~Hz}$ on each side of an Arrow-shape barrier could improve the mean broadband insertion loss of the barrier at nine different receiver positions above the ground by $2.3 \mathrm{~dB}(\mathrm{~A})$. The amount of improvement in mean insertion loss with a $400 \mathrm{~Hz}$ tuned QRD T-shape barrier compare to an equivalent absorbent T-shape barrier was found to be $0.9 \mathrm{~dB}(\mathrm{~A})$. In contrast, using QRDs on cylindrical top barrier was found to decrease the A-weighted performance of the absorbent profile barrier by $1 \mathrm{~dB}(\mathrm{~A})$.

vi. Reducing the well width of utilised QRD on T- shape profile barriers reduces the overall A-weighted insertion loss of the barriers. 
vii. Employing QRD with less prime number resulted in fewer frequency peaks in the insertion loss spectrum, although the overall barrier performance is slightly higher than model "D” with the same frequency design but smaller well width.

viii. The QRD covered T-shape barrier (model “G”) was found to be effective in both the far field at distance larger than $10 \mathrm{~m}$ from the barrier and areas close to the ground. The efficiency of model "G" is less than its equivalent absorbent barrier at the areas close to the barrier above $3 \mathrm{~m}$ height. For the Arrow - shape QRD design the insertion loss at areas close to the ground could be less than that of its equivalent absorptive barrier.

It should be noted that the above results were obtained purely by numerical simulations. Although the boundary element method used for the simulation has been found in previous studies to have very good accuracy when applied to rigid and absorptive, and reactive profiled barriers [1,2,4] and to QRD at normal incidence [10], its accuracy when dealing with QRD on a barrier has yet to be confirmed with measurements. Furthermore the numerical models assume that the admittance distribution on the QRD top surface is given by the simple plane wave admittance of the well sequence. This assumption will break down at frequencies above the upper cut-off frequency of the QRD as shown in Fig. 4. The viscous and thermal losses in the wells will also complicate the admittance especially when the well width is small. Besides, environmental factors such as atmospheric turbulence may further limit the increase in insertion loss that could be obtained by the application of the QRD. Further work is being carried out to investigate the above and will be subjects of future papers. 
Table 1. Design model names and corresponding configurations.

\begin{tabular}{c|ccccccc}
\hline Models & $\begin{array}{c}\text { Cross } \\
\text { section }\end{array}$ & $\begin{array}{c}\text { No. Of } \\
\text { QRD }\end{array}$ & $\begin{array}{c}\text { N } \\
\text { Frequency } \\
f_{r}(\mathrm{kHz})\end{array}$ & $\begin{array}{c}\text { Well } \\
\text { width } \\
w(\mathrm{~cm})\end{array}$ & Sequence & Description \\
\hline $\mathrm{A}$ & $\mathrm{T}$ & 1 & 7 & 1 & 12 & 1 & - \\
$\mathrm{B}$ & $\mathrm{T}$ & 2 & 7 & 1 & 6 & 11 & - \\
$\mathrm{C}$ & $\mathrm{T}$ & 6 & 7 & 1 & 2 & $11111-1$ & - \\
$\mathrm{D}$ & $\mathrm{T}$ & 1 & 7 & 0.5 & 12 & 1 & - \\
$\mathrm{E}$ & $\mathrm{T}$ & 2 & 7 & 0.5 & 6 & 11 & - \\
$\mathrm{F}$ & $\mathrm{T}$ & 6 & 7 & 0.5 & 2 & $11111-1$ & - \\
$\mathrm{G}$ & $\mathrm{T}$ & 1 & 7 & 0.4 & 12 & 1 & - \\
$\mathrm{H}$ & $\mathrm{T}$ & 2 & 7 & 0.4 & 6 & 11 & - \\
$\mathrm{I}$ & $\mathrm{T}$ & 6 & 7 & 0.4 & 2 & $11111-1$ & - \\
$\mathrm{K}$ & $\mathrm{T}$ & 1 & 5 & 0.5 & 18 & 1 & - \\
$\mathrm{L}$ & Arrow & 2 & 7 & 0.5 & 12 & 11 & $* \theta=60^{\circ}$ \\
$\mathrm{M}$ & Arrow & 2 & 7 & 0.4 & 12 & 11 & $\theta=60^{\circ}$ \\
$\mathrm{N}$ & Arrow & 4 & 7 & 0.4 & 12 & $11 \& 11$ & Top \& \\
$\mathrm{O}$ & Cylinder & 2 & 7 & 0.4 & 12 & 11 & underneath \\
$\mathrm{R}$ & $\mathrm{Y}$ & 2 & 7 & 0.4 & 12 & 11 & $\theta=30^{\circ}$ \\
$\mathrm{S}$ & $\mathrm{Y}$ & 2 & 7 & 0.4 & 12 & 11 & $\theta=60^{\circ}$ \\
\hline
\end{tabular}

* $\theta$ is the angle for the Arrow and $\mathrm{Y}$ - shape barrier described in Fig.2. 
Table 2. Broadband A-weighted mean insertion loss at nine receivers for various forms of barriers. A typical traffic loss spectrum is assumed.

\begin{tabular}{|l|c|c|}
\hline & \multicolumn{2}{|c|}{ Broad band IL over 9 receivers (dB(A)) } \\
\hline Barrier type & Mean & $\Delta \mathrm{L}$ \\
\hline T- shape & 16.9 & -1.2 \\
\hline Cylinder & 13.8 & -4.3 \\
\hline Arrow & 14.7 & -3.4 \\
\hline Y- shape & 17.1 & -1.0 \\
\hline "Ref" (AT- shape) & 18.1 & 0.0 \\
\hline A cylinder & 15.5 & -2.6 \\
\hline A Arrow & 17.2 & -0.9 \\
\hline A Y- shape & 18.8 & 0.7 \\
\hline Model A & 16.6 & -1.6 \\
\hline Model B & 16.3 & -1.8 \\
\hline Model C & 16.8 & -1.3 \\
\hline Model D & 17.7 & -0.4 \\
\hline Model E & 16.7 & -1.4 \\
\hline Model F & 16.0 & -2.1 \\
\hline Model G & 19.0 & 0.9 \\
\hline Model H & 18.3 & 0.1 \\
\hline Model I & 16.6 & -1.6 \\
\hline Model K & 17.9 & -0.2 \\
\hline Model M & 17.0 & -1.2 \\
\hline Model O & 14.5 & -3.6 \\
\hline Model R & 18.8 & 0.7 \\
\hline Model S & 19.0 & 0.9 \\
\hline & & \\
\hline
\end{tabular}

Receiver positions are: 1 at $(-20.0) ; 2$ at $(-50,0) ; 3$ at $(-100,0) ; 4$ at $(-20,1.5) ; 5$ at $(-50,1.5) ; 6$ at $(-100,1.5) ; 7$ at $(-20,3)$; 8 at $(-$ $50,3) ; 9$ at $(-100,3)$ and $\Delta \mathrm{IL}$ is the difference between the mean insertion loss for a mentioned barrier and the mean insertion loss for an absorbent T-Shape screen. Source position is at $(5,0)$. For the absorptive cases (types with prefix "A") the upper surface of the cap is covered with an absorbent material with $\sigma=20000$ Rayl (MKS) and thickness of $0.2445 \mathrm{~m}$. QRD barrier types are as described in Table 1. 
Table. 3. A comparison of broadband A- weighted mean insertion loss at three different receiver points on the ground for each barrier type having different top coverings: rigid, absorptive and QRD. (a) T- shape, (b) Cylinder- shape (c) Arrow- shape, (d) Y-shape.

(a)

\begin{tabular}{|c|c|c|c|c|c|c|}
\hline \multirow{2}{*}{$\begin{array}{c}\text { Distance from } \\
\text { receiver }(\mathrm{m})\end{array}$} & \multicolumn{2}{|c|}{$\mathrm{T}$ - shape } & \multicolumn{2}{c|}{$\mathrm{A} T$ - shape } & \multicolumn{2}{c|}{ QRD edged (Model G) } \\
\cline { 2 - 7 } & Mean $(\mathrm{dB}(\mathrm{A}))$ & $\Delta \mathrm{lL}(\mathrm{dB}(\mathrm{A}))$ & Mean $(\mathrm{dB}(\mathrm{A}))$ & $\Delta \mathrm{lL}(\mathrm{dB}(\mathrm{A}))$ & Mean $(\mathrm{dB}(\mathrm{A}))$ & $\Delta \mathrm{lL}(\mathrm{dB}(\mathrm{A}))$ \\
\hline 20 & 15.9 & 0.0 & 17.2 & 1.4 & 18.2 & 2.3 \\
\hline 50 & 15.1 & 0.0 & 16.5 & 1.4 & 17.5 & 2.4 \\
\hline 100 & 14.9 & 0.0 & 16.2 & 1.4 & 17.3 & 2.4 \\
\hline
\end{tabular}

(b)

\begin{tabular}{|c|c|c|c|c|c|c|}
\hline \multirow{2}{*}{$\begin{array}{c}\text { Distance from } \\
\text { receiver }(\mathrm{m})\end{array}$} & \multicolumn{2}{|c|}{ Cylinder - shape } & \multicolumn{2}{c|}{ A Cylinder - shape } & \multicolumn{2}{c|}{ QRD edged (Model 0) } \\
\cline { 2 - 6 } & Mean $(\mathrm{dB}(\mathrm{A}))$ & $\Delta \mathrm{lL}(\mathrm{dB}(\mathrm{A}))$ & Mean $(\mathrm{dB}(\mathrm{A}))$ & $\Delta \mathrm{lL}(\mathrm{dB}(\mathrm{A}))$ & Mean $(\mathrm{dB}(\mathrm{A}))$ & $\Delta \mathrm{lL}(\mathrm{dB}(\mathrm{A}))$ \\
\hline 20 & 13.6 & 0.0 & 14.6 & 1.1 & 13.2 & -0.4 \\
\hline 50 & 11.1 & 0.0 & 13.1 & 2.0 & 12.5 & 1.4 \\
\hline 100 & 11.0 & 0.0 & 12.9 & 1.9 & 12.4 & 1.4 \\
\hline
\end{tabular}

(c)

\begin{tabular}{|c|c|c|c|c|c|c|}
\hline \multirow{2}{*}{$\begin{array}{c}\text { Distance from } \\
\text { receiver }(\mathrm{m})\end{array}$} & \multicolumn{2}{|c|}{ Arrow - shape } & \multicolumn{2}{c|}{ A Arrow - shape } & \multicolumn{2}{c|}{ QRD edged (Model M) } \\
\cline { 2 - 7 } & Mean (dB(A)) & $\Delta \mathrm{LL}(\mathrm{dB}(\mathrm{A}))$ & Mean (dB(A)) & $\Delta \mathrm{LL}(\mathrm{dB}(\mathrm{A}))$ & Mean (dB(A)) & $\Delta \mathrm{lL}(\mathrm{dB}(\mathrm{A}))$ \\
\hline 20 & 13.4 & 0.0 & 16.5 & 3.1 & 16.3 & 2.9 \\
\hline 50 & 12.9 & 0.0 & 15.8 & 2.9 & 15.8 & 2.9 \\
\hline 100 & 12.8 & 0.0 & 15.6 & 2.8 & 15.7 & 2.9 \\
\hline
\end{tabular}

(d)

\begin{tabular}{|c|c|c|c|c|c|c|}
\hline \multirow{2}{*}{$\begin{array}{c}\text { Distance from } \\
\text { receiver }(\mathrm{m})\end{array}$} & \multicolumn{2}{|c|}{$\mathrm{Y}$ - shape } & \multicolumn{2}{c|}{$\mathrm{A} Y$ - shape } & \multicolumn{2}{c|}{ QRD edged (Model S) } \\
\cline { 2 - 7 } & Mean (dB(A)) & $\Delta \mathrm{IL}(\mathrm{dB}(\mathrm{A}))$ & Mean (dB(A)) & $\Delta \mathrm{IL}(\mathrm{dB}(\mathrm{A}))$ & Mean (dB(A)) & $\Delta \mathrm{IL}(\mathrm{dB}(\mathrm{A}))$ \\
\hline 20 & 16.3 & 0.0 & 18.3 & 2.1 & 18.4 & 2.1 \\
\hline 50 & 15.2 & 0.0 & 17.1 & 1.9 & 17.2 & 2.0 \\
\hline 100 & 15.0 & 0.0 & 16.8 & 1.8 & 17.0 & 2.1 \\
\hline
\end{tabular}

NB: $\Delta \mathrm{IL}$ is the difference between the mean insertion loss for a mentioned barrier and the mean insertion loss of its equivalent rigid barrier. 


\section{References}

1. D.C. Hothersall, D.H. Crombie, and S.N. Chandler-Wilde, "The performance of Tshape profile and associated noise barriers”, Applied Acoustics 32(4), pp.269-281, 1991.

2. D.H. Crombie, D.C. Hothersall, and S.N. Chandler-Wild, "Multiple-edge noise barrier”, Applied Acoustics 44(4), pp.353-367, 1995.

3. P. Menounou, I.J. Busch-Vishniac, D. T. Blackstock, "Directive line source model: A new model for sound diffraction by half planes and wedges", J. Acoust. Soc. Am. 107 (6), pp.2973-2986, 2000.

4. Kyoji Fujiwara, David C. Hothersall and Chul-hwan Kim, "Noise barriers with reactive surfaces”, Applied Acoustics, 53 (4), pp. 225-272, 1998.

5. R. Seznec, "Diffraction of sound around barriers: use of boundary elements technique”, Journal of Sound Vibration 73(2), pp.195-209, 1980

6. D. C. Hothersall, S. N. Chandler-Wilde and M. N. Hajmirzae, "Efficiency of single noise barriers”, Journal of Sound Vibration, 146(2), pp.303-322, 1991.

7. M. E. Delany and E. N. Bazely, "Acoustical properties of fibrous absorbent material”, Applied Acoustics 3(2), pp.105-116, 1970.

8. Y. W. Lam and S. C. Roberts, "A simple method for accurate prediction of finite barrier insertion loss”, J.Acous.Soc.Am. 93, 1445-1452, 1993.

9. H. Kuttruff, "Sound absorption by psedostochastic diffusers (Schroeder diffusers)", Applied Acoustics 42(3), pp.215-231, 1994.

10. Trevor J. Cox and Y. W. Lam, "Prediction and evaluation of the scattering from quadratic residue diffusers”, J. Acoust. Soc. Am. 95 (1), pp.297-305, 1994.

11. K. Fujiwara and T. Miyajima, "Absorption characristics of practically constructed Schroeder diffuser of quadratic-residue type”, Applied Acoustics 35(2), pp.149-152, 1992.

12. T. Wu, T. J. Cox, and Y. W. Lam, "From a profiled diffuser to an optimised absorber”, J. Acoust. Soc. Am . 108 (2), pp.643-650, 2000.

13. BS EN 1793-3:1998, Road traffic noise reducing devices- Test method for determining the acoustic performance, Part 3. Normalized traffic noise spectrum. 
Figure Captions:

Figure 1. The two dimensional model.

Figure 2. Cross-sections of Barriers with Different Top Profiles.

Figure 3. Dimensions of the T- shape barrier having a QRD ( $\mathrm{N}=7$ and $\left.f_{r}=1 \mathrm{kHz}\right)$.

Figure 4. Comparison between two prediction methods for model "G" at receiver point ($50,0)$.

Figure 5. Results of employing QRD tuned to $400 \mathrm{~Hz}$ on different barrier profiles at the receiver point (-50,0): with (a) T-shape (b) Cylinder - shape (c) Arrow-shape (d) Y-shape. Source is at $(5,0)$.

Figure 6. Predicted frequency spectra of barrier insertion loss for ten different QRD T-shape barriers at the receiver point $(-50,0)$, with top surfaces having: (a) 1,2 \& 6 QRDs (N=7 \& $f_{r}$ $=1 \mathrm{kHz}$ ), (b) 1,2 \& 6 QRDs ( $\left.=7 \& f_{r}=500 \mathrm{kHz}\right)$, (c) $1,2 \& 6$ QRDs $\left(\mathrm{N}=7 \& f_{r}=400 \mathrm{kHz}\right)$ and (d) a QRD (N=5 \& $\left.f_{r}=1 \mathrm{kHz}\right)$. Well widths of the QRD changes within each subplot according to Table 1.

Figure 7. Predicted spectra of Insertion Loss for QRD Y- Shape barriers along with T- Shape barrier at receiver point $(-50,0)$.

Figure 8. The effect of QRD covers with different frequency designs compared with an absorptive cover on a $\mathrm{T}$ - shape barrier at the receiver point $(-50,0)$.

Figure 9. A comparison between T- shape QRD barrier (Model “G”) and an Arrow - shape QRD barrier (Model "M") with the "Ref” model at the receiver point $(-50,0)$. The QRDs used are of the same design in both QRD barriers.

Figure 10. Differences in Insertion Loss of two QRD barrier models with the same QRD but different top profiles relative to that of the "Ref" model barrier at the receiver point $(-50,0)$.

Figure 11. Differences of Insertion Loss of three QRD barrier models with different frequency designs relative to that of the "Ref" model barrier at receiver point $(-50,0)$.

Figure 12. Contour plot of differences of broad band A- weighted insertion loss of barrier model "G" relative to "Ref" at 400 receiver positions.

Figure 13. Contour plot of differences of broad band A- weighted insertion loss of barrier model "M" relative to its equivalent Arrow - shape barrier at 400 receiver positions. 


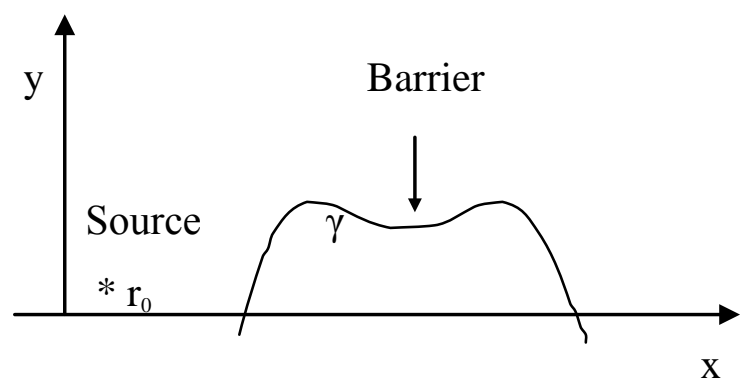

Figure 1. The two dimensional model 


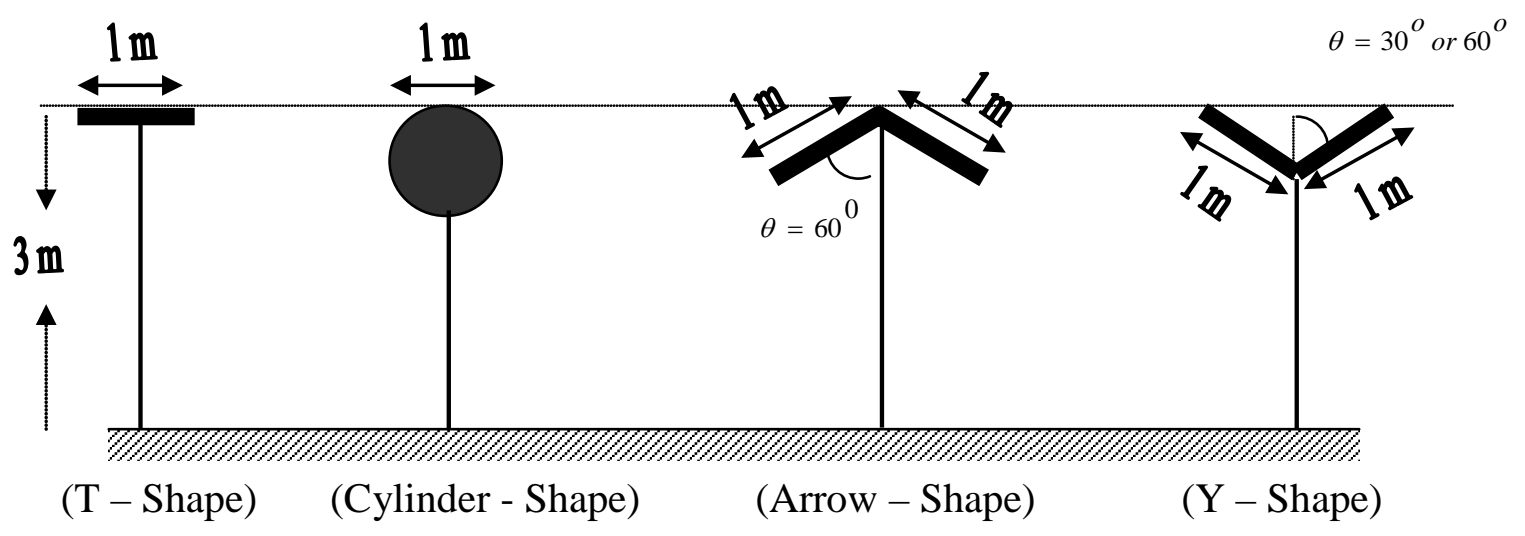

Figure 2. Cross-sections of Barriers with Different Top Profiles. 


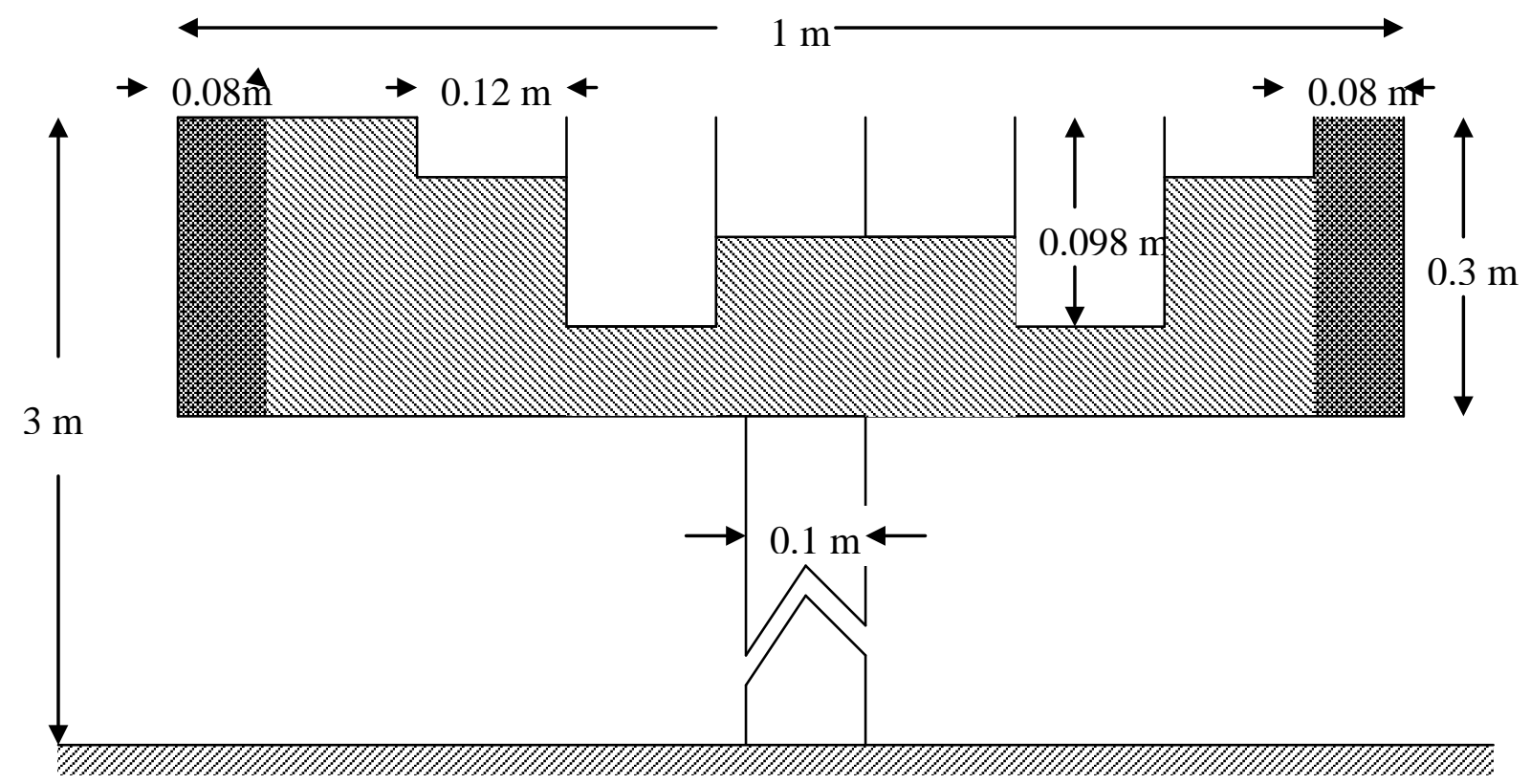

Figure 3. Dimensions of the T- shape barrier having a QRD ( $\mathrm{N}=7$ and $\left.f_{r}=1 \mathrm{kHz}\right)$. 


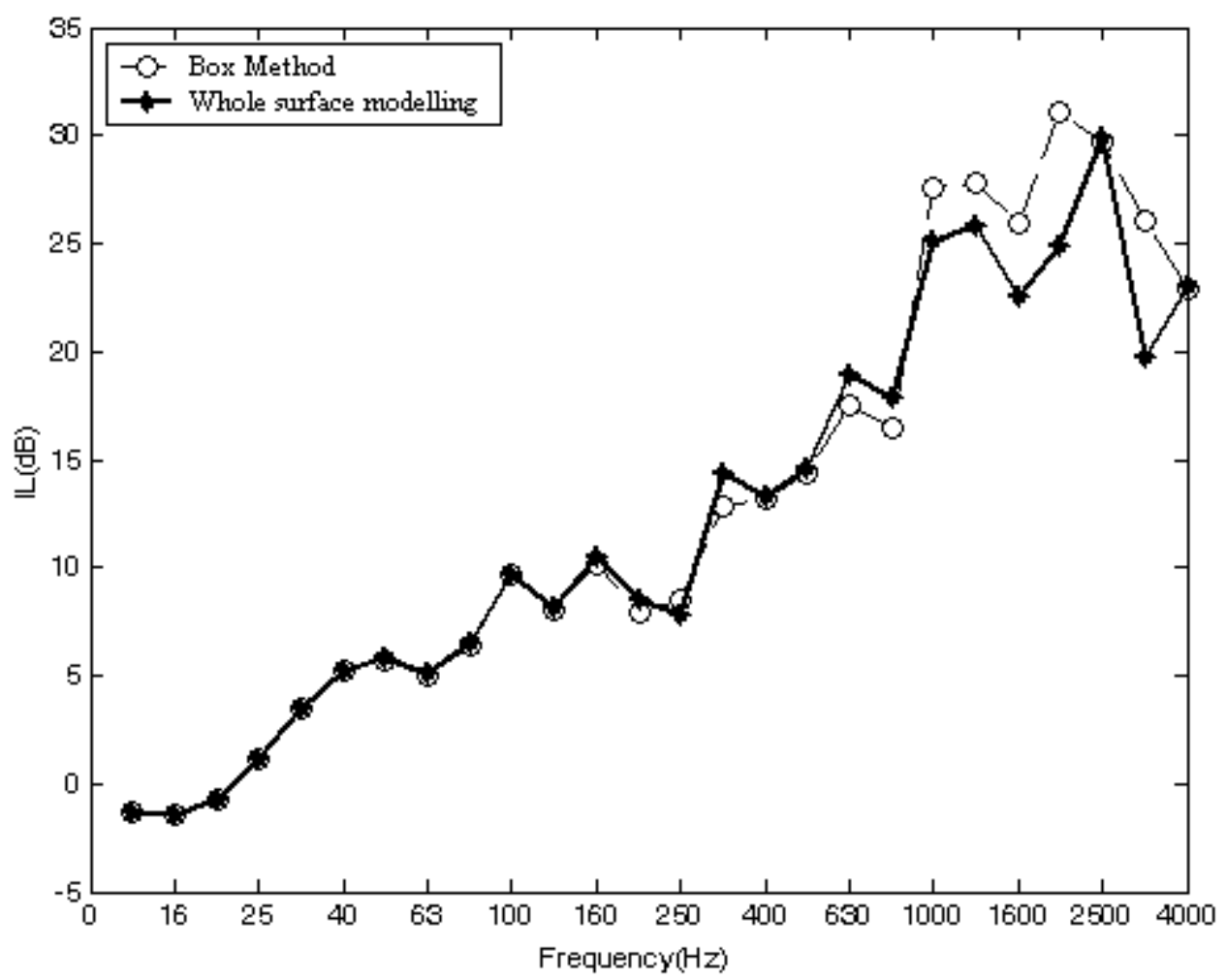

Figure 4. Comparison between two prediction methods for model " $G$ " at receiver point ($50,0)$ 
(a)

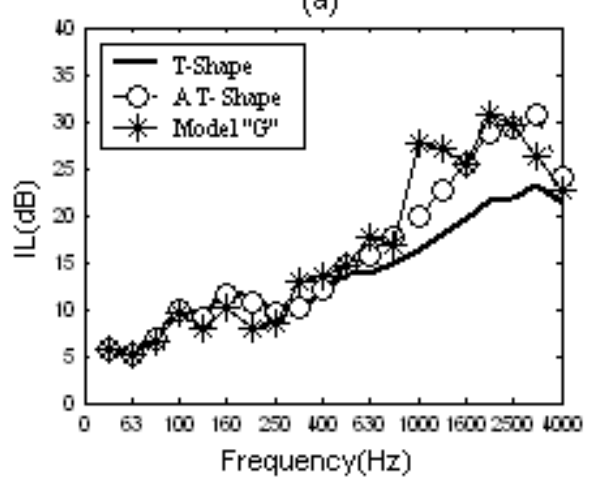

(c)

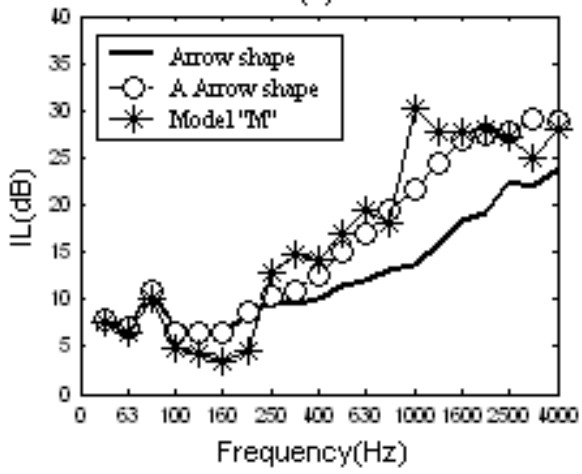

(b)

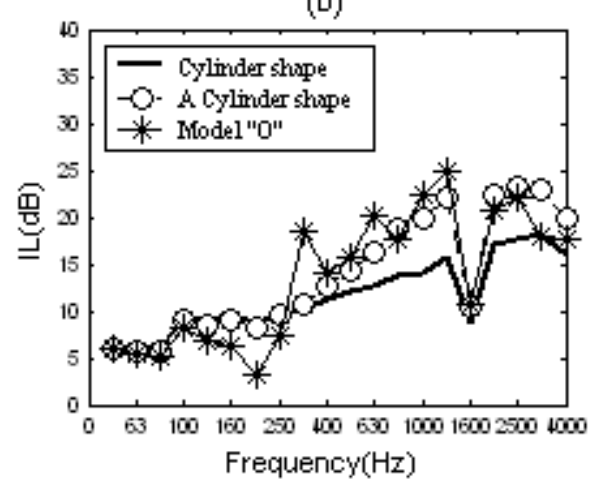

(d)

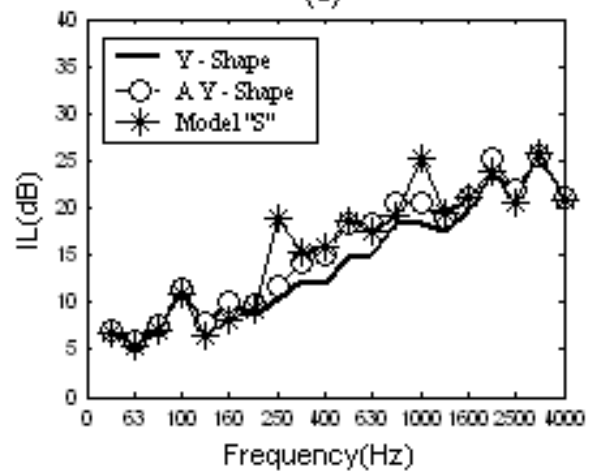

Figure 5. Results of employing QRD tuned to $400 \mathrm{~Hz}$ on different barrier profiles at the receiver point (-50,0): with (a) T-shape (b) Cylinder - shape (c) Arrow-shape (d) Y- shape. Source is at $(5,0)$. 
(a)

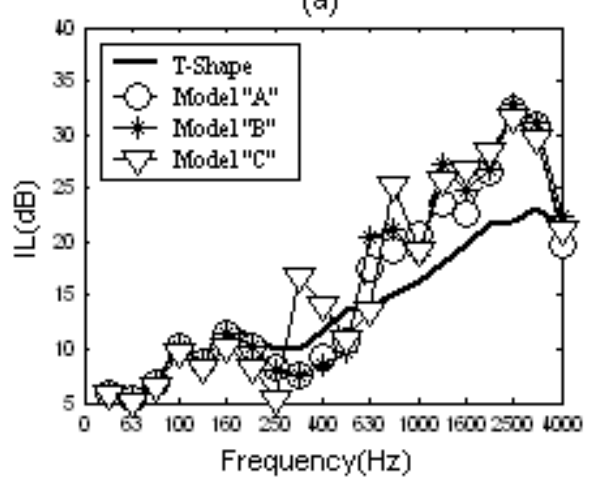

(c)

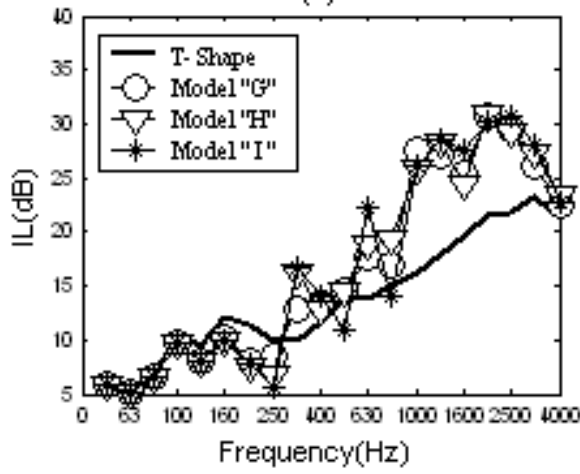

(b)

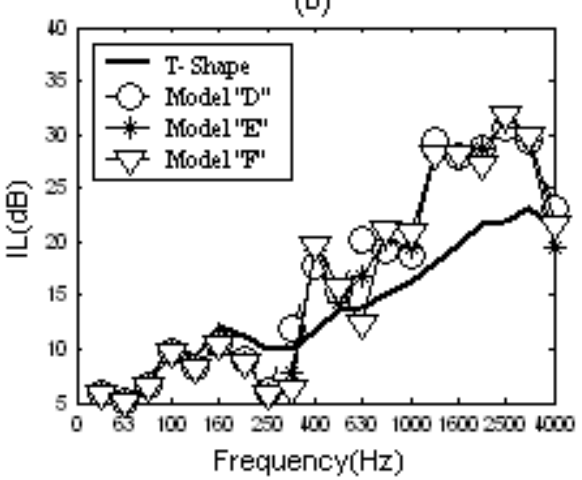

(d)

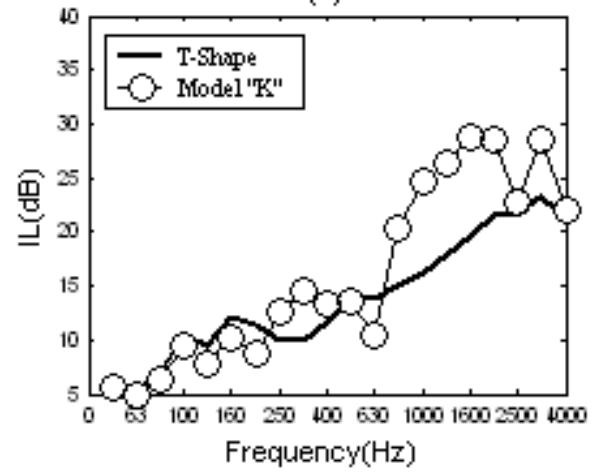

Figure 6. Predicted frequency spectra of barrier insertion loss for ten different QRD T-shape barriers at the receiver point $(-50,0)$, with top surfaces having: (a) 1,2 \& 6 QRDs ( $N=7 \& f_{r}=1 \mathrm{kHz}$ ), (b) 1,2 \& 6 QRDs ( $\left.\mathrm{N}=7 \& f_{r}=500 \mathrm{kHz}\right),\left(\right.$ c) $1,2 \& 6 \mathrm{QRDs}\left(\mathrm{N}=7 \& f_{r}=400 \mathrm{kHz}\right)$ and (d) a $\mathrm{QRD}\left(\mathrm{N}=5 \& f_{r}=\right.$ $1 \mathrm{kHz}$ ). Well widths of the QRD changes within each subplot according to Table 1. 


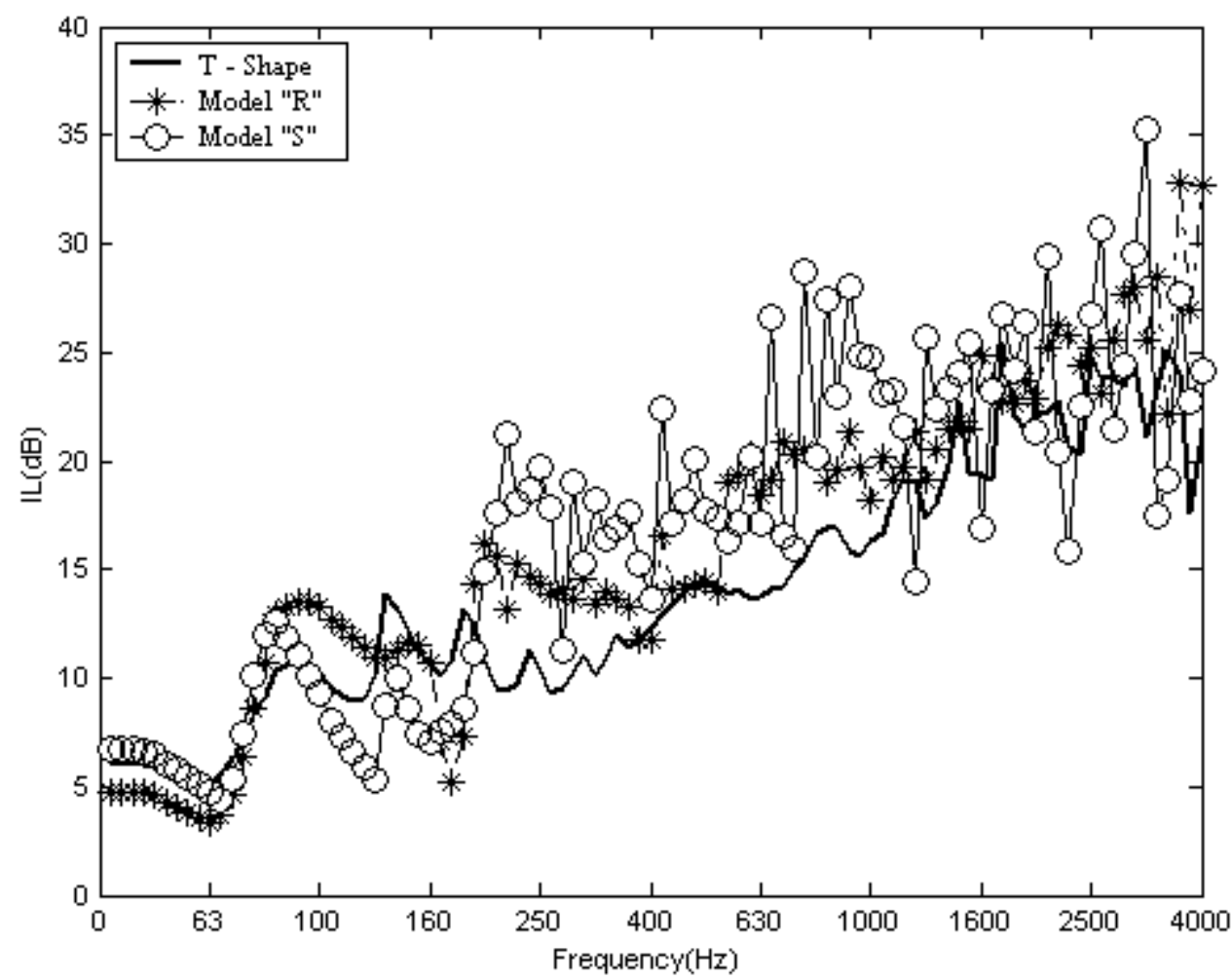

Figure 7. Predicted spectra of Insertion Loss for QRD Y- shape barriers along with T- shape barrier at receiver point $(-50,0)$. 


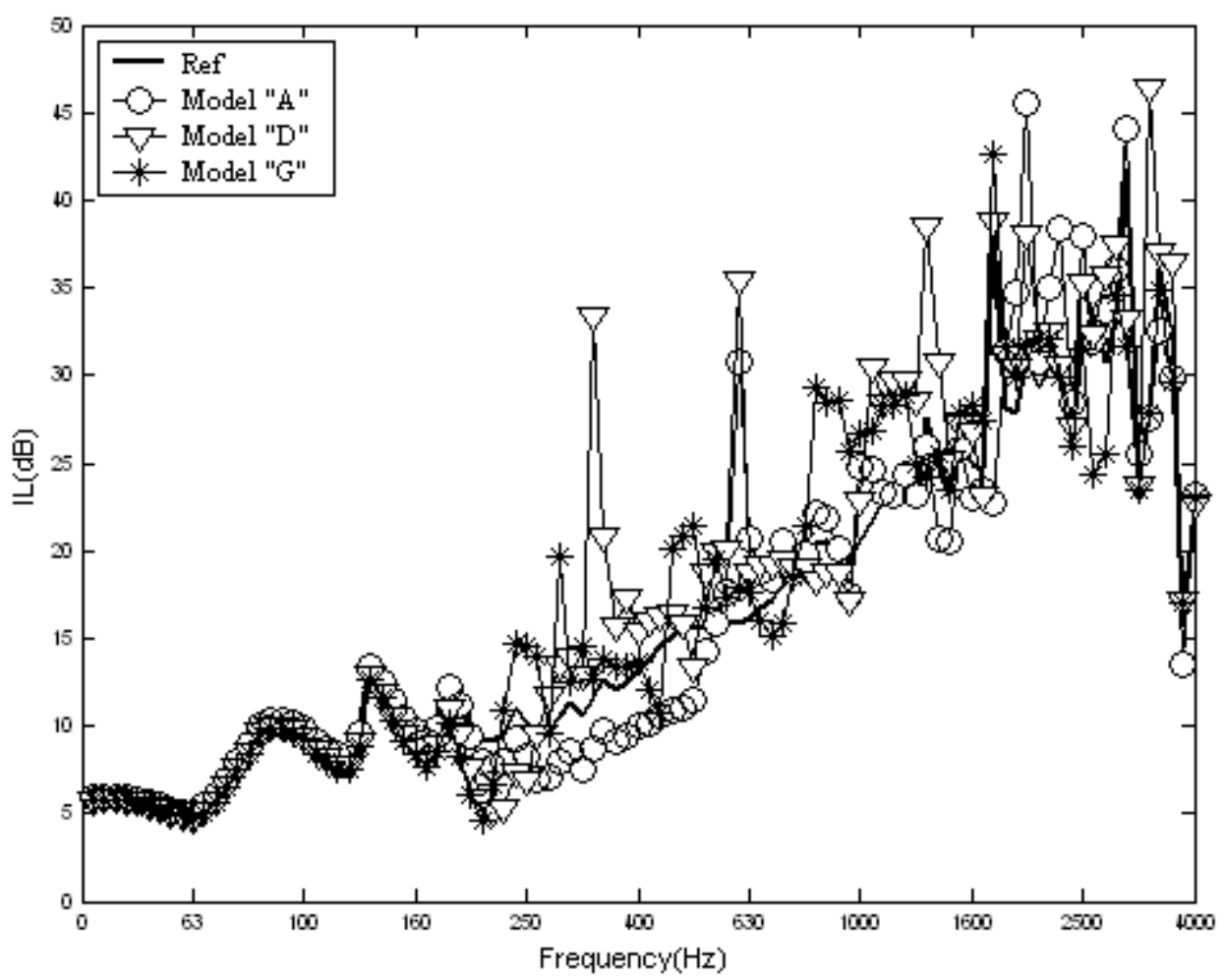

Figure 8. The effect of QRD covers with different frequency designs compared with an absorptive cover on a T-shape barrier at the receiver point $(-50,0)$. 


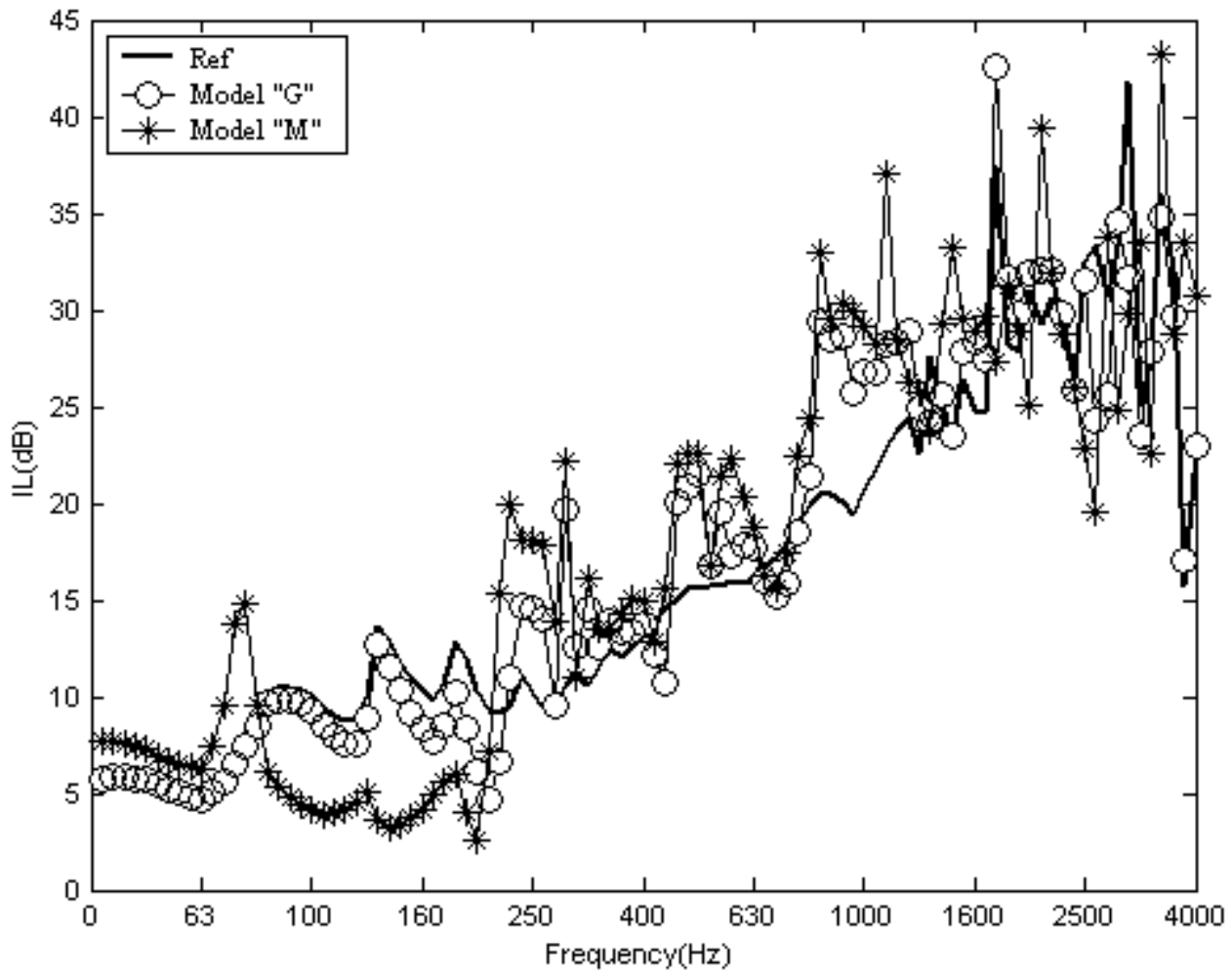

Figure 9. A comparison between T- shape QRD barrier (Model “G”) and an Arrow - shape QRD barrier (Model "M") with the "Ref” model at the receiver point (-50,0). The QRDs used are of the same design in both QRD barriers. 


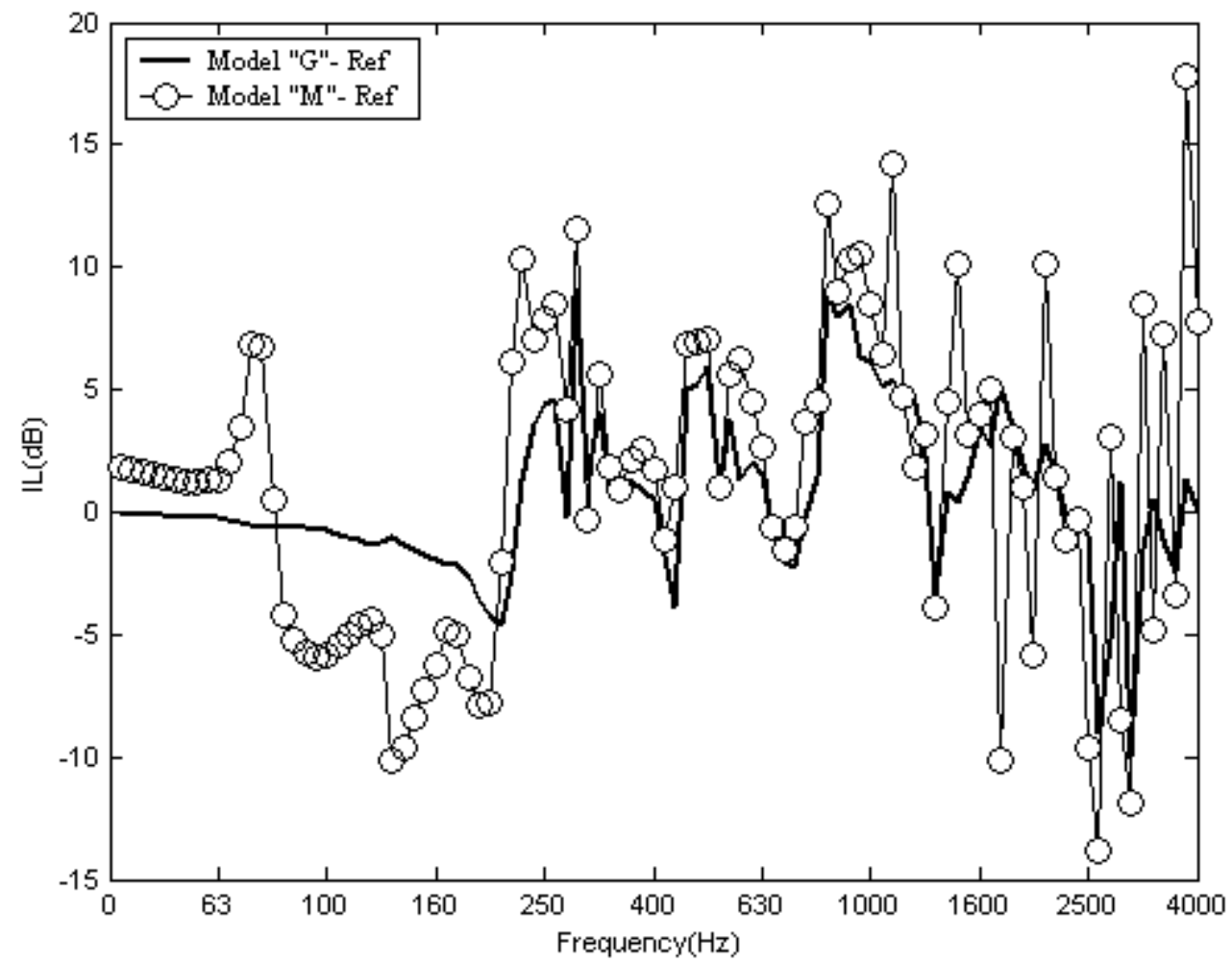

Figure 10. Differences in Insertion Loss of two QRD barrier models with the same QRD but different top profiles relative to that of the "Ref" model barrier at the receiver point $(-50,0)$. 


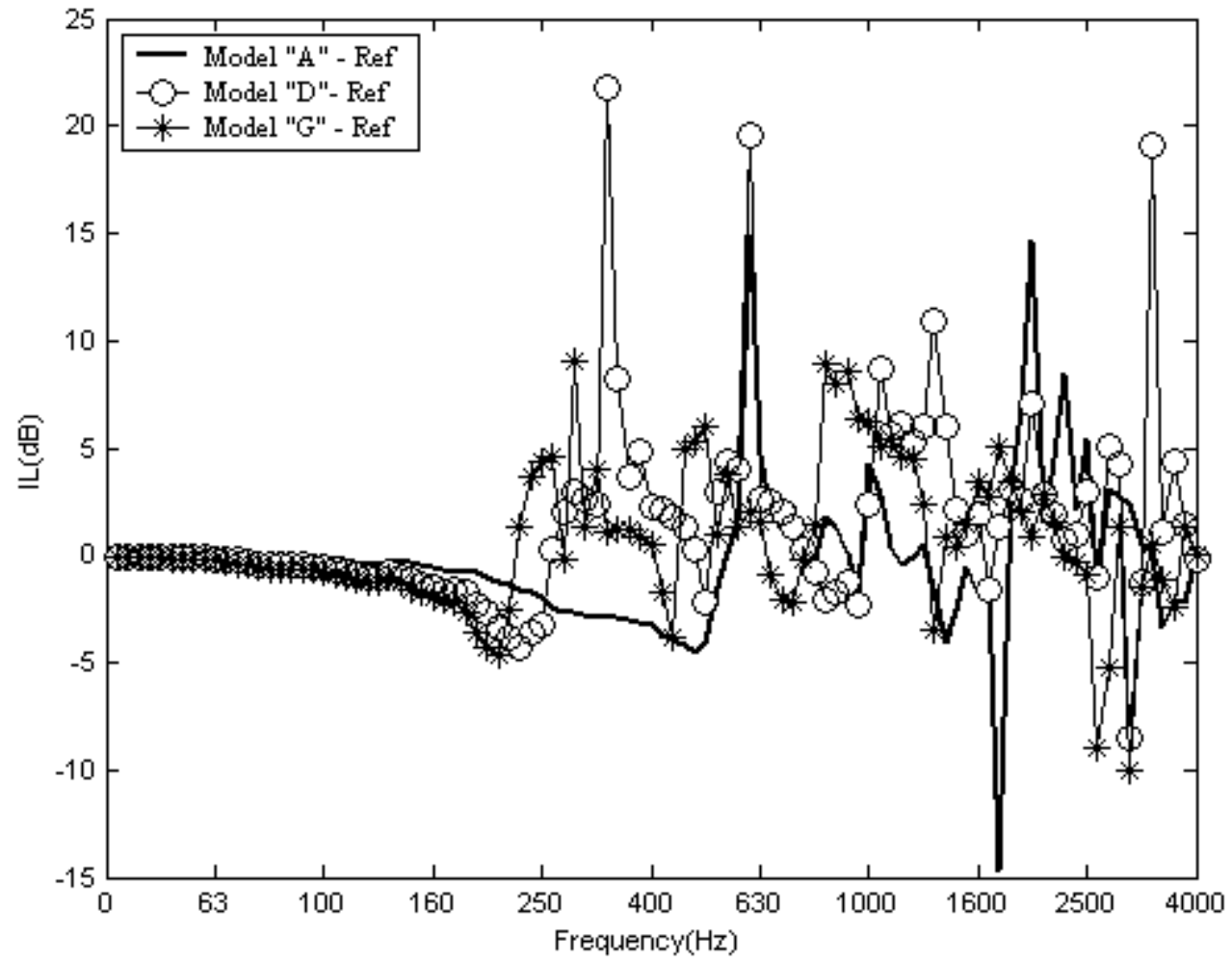

Figure 11. Differences of Insertion Loss of three QRD barrier models with different frequency designs relative to that of the "Ref" model barrier at receiver point $(-50,0)$. 


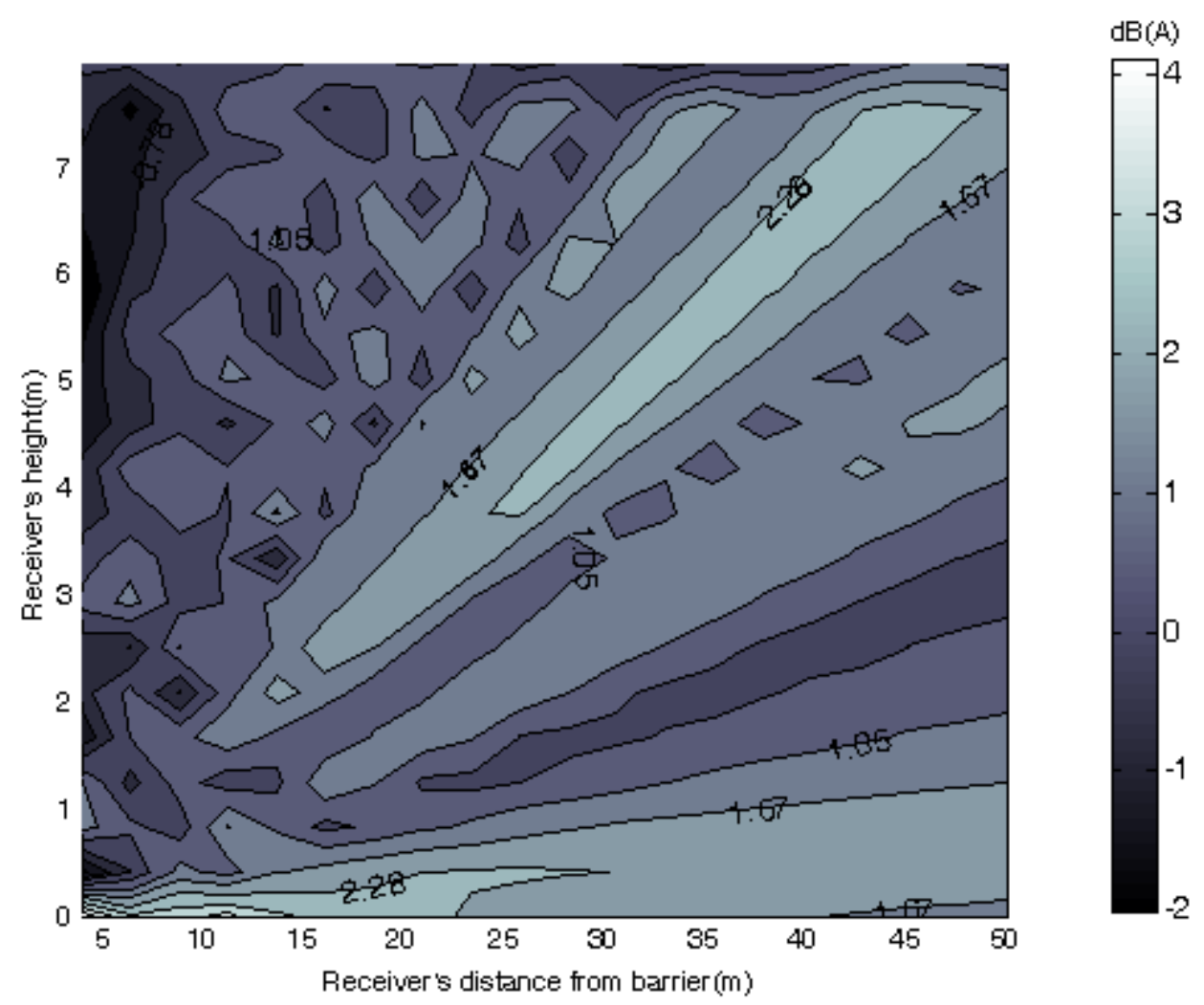

Figure 12. Contour plot of differences of broad band A- weighted insertion loss of barrier model "G” relative to "Ref” at 400 receiver positions. 


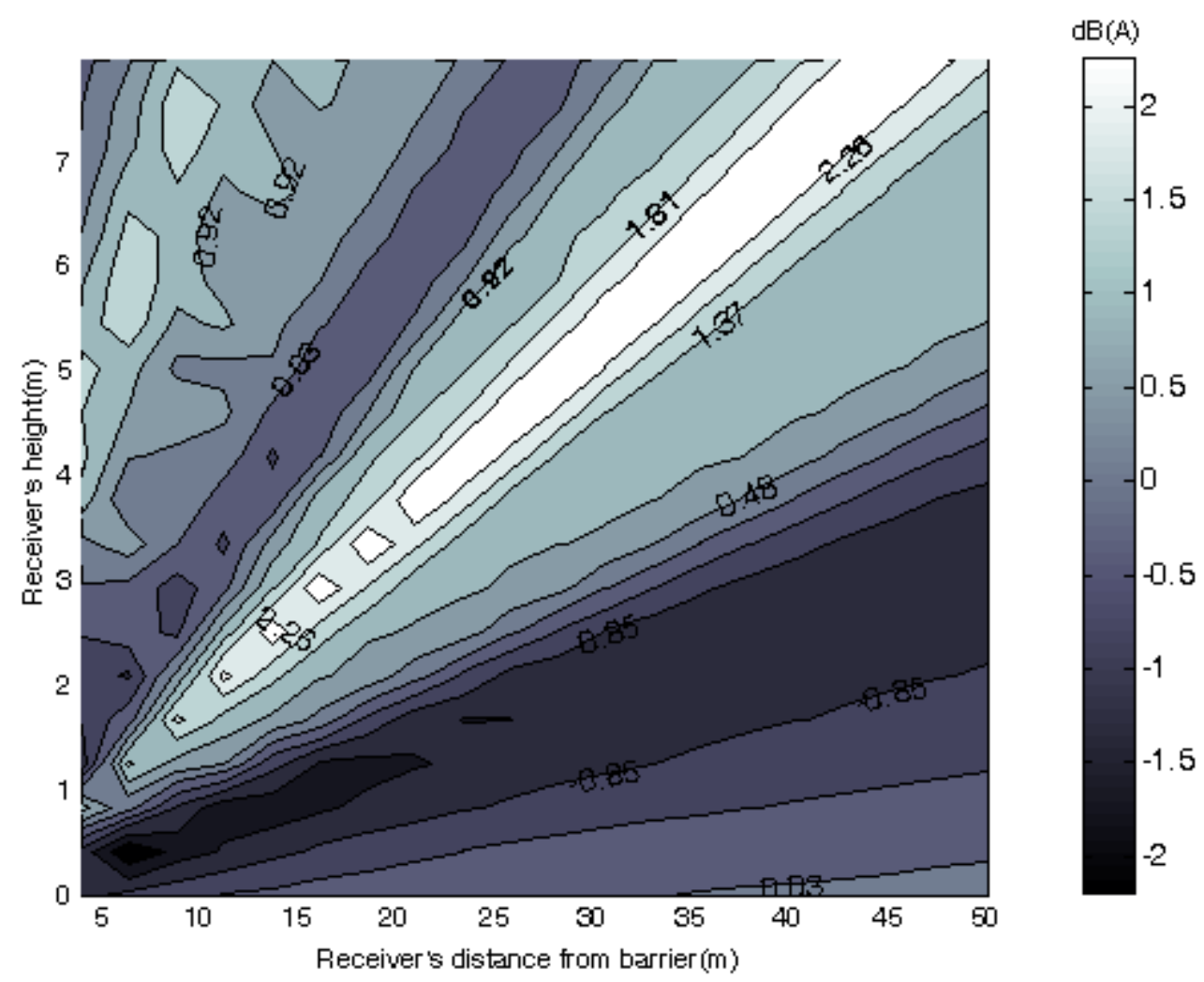

Figure 13. Contour plot of differences of broad band A- weighted insertion loss of barrier model " $M$ " relative to its equivalent absorbent Arrow - shape barrier at 400 receiver positions. 